\title{
Efficacy and Safety of Nasal Administration of "Na- no-L-DOPA” Based on PLGA Nanoparticles
}

\author{
I. G. Kondrasheva ${ }^{1}$, T. A. Antipova ${ }^{1}$, G. G. Barsegyan ${ }^{1}$, P. Ye. Gambaryan' ${ }^{2}$ A. A. Guseva ${ }^{2}$, A. A. Kamensky ${ }^{2}$ \\ ${ }^{1}$ Research Center of Molecular Diagnostics \& Therapy, Moscow, Russia \\ ${ }^{2}$ Moscow State University, Moscow, Russia \\ Email: kondrashevl@list.ru
}

Received 2012

\begin{abstract}
Inclusion of $L-D O P A$, the standard Parkinson's disease medication, into polymeric particles (PLGA) results in optimization the drug metabolism and increasing its bioavailability, significantly increases of physical endurance, better coordination and lower anxiety in Wistar rats, when chronically administered nasally.
\end{abstract}

Keywords: Nanopaticls; L-DOPA; Nasal Administration; Parkinson’s Disease

\section{Introduction}

Nasal administration of medications is a well known and currently widely applicable method of administration of a number of broad-spectrum drugs, both locally and systemically acting, the interest in which, as a non-invasive method of administration drugs, deepens on [1]. Due to vascular plexuses in nasal cavity and a large absorption area, nasal administration of medications becomes a prospective method of delivering drugs directly into the blood stream, promoting fast achievement of the effective drug level in blood. Additional benefits of nasal administration are ease of performing, usability for patients, and a relatively low cost. Moreover, drugs can be transported from nasal cavity into the central nervous system (CNS) without involving the blood circulatory system of nasal-cavity lining mucosa, by extracellular way - along the trifacial nerve and the olfactory nerve. Olfactory nerve sheathes do not have blood-brain barriers, and the drug can directly enter the brain [2-5]. This fact is the focus of attention, since it provides new opportunities in treating CNS diseases, particularly neurodegenerative brain diseases, such as Parkinson's disease or Alzheimer's disease [6]. The "nose-brain" way leads to practically immediate delivery of some drugs into the cerebrospinal fluid, bypassing blood and the blood-brain barrier (BBB) [7-10]. L-3,4-dihydroxyphenylalanine (L-DOPA) is the precursor of dopamine that is responsible for the central mechanism of motion control and coordination. Applying L-DOPA in the treatment of Parkinson's disease has a number of disadvantages. Optimizing the drug metabolism and increasing its bioavailability and potency are possible, if it is included into nanoparticles based on poly(lactic-co-glycolic acid) (PLGA), a biodegradable and non-toxic polymer. Including a drug into the particles allows modulating the biopharmaceutical properties of the medications administered, their bioavailability and, as a consequence, their potency [11]. Moreover, nanocontainers protect the drug components against chemical/enzymatic degradation, provide durable release, decrease the drug toxicity for peripheral organs, and represent drug containers suitable for treating the CNS diseases [12].

The purpose of this research is the estimation of efficacy and safety of the nasal administration of L-DOPA, the precursor of the dopamine neurotransmitter, in form of a nanoparticle medication ("Nano-L-DOPA") developed on the basis of poly(lactic-co-glycolic acid) (PLGA) for modulating (increasing) the exercise performance of rats Wistar, as compared to the efficacy and safety of L-DOPA, the initial standard medication.

\section{Materials and Methods}

In this paper, L-DOPA (Sigma, China), $1 \mathrm{mg} / \mathrm{kg}$, and "Nano-LDOPA” (Research Center of Molecular Diagnostics \& Therapy, Russia), $1 \mathrm{mg} / \mathrm{kg}, 5$ and $10 \mathrm{mg} / \mathrm{kg}$ (by L-DOPA), were researched. Particle sizes of the latter one varied from 250 through $400 \mathrm{~nm}$, while the L-DOPA substance content within the "Nano-L-DOPA" composition ranged from 9.5 through $12.0 \%$ of the mass. The research in the dynamics of the drug release from the nanocomposition in a model experiment in vitro testifies that up to $70 \%$ of L-DOPA contained in the particles are released within the first 30 minutes, while the resting $30 \%$ of the active substance are released within the subsequent 10 hours. The solutions of L-DOPA were prepared immediately prior to the start of the experiment. Normal saline solution was used as a dissolution medium, ascorbic acid (1\%, pH 2.6) being added for bettering the dissolubility and preventing the drug from oxidation on dissolving. Animals in the Baseline were administered with blank particles in the amount corresponding with the maximum dose of the nano-medication. In the research, male Wistar rats weighing 200-250 g and BALB/c mice (20 g) were used.

The drug influence upon the exercise performance, the motion activity and the behavior of the laboratory animals in the long-term experiment was researched comprehensively using a number of tests.

1) In the dynamic load conditions, in test "Forced Swimming” up to complete fatigue with weights $(15 \%$ of their body masses), the drug influence upon the exercise performance of 
the rodents was estimated for the mixed-nature loads (the limits of anaerobic and aerobic power). The animals were placed into water-containing tanks where the duration of their active and passive swimming was registered. The fatigue criterion was the animal's sinking to the tank bottom, upon which it did not rise within 10 seconds. The drug potency criterion was a positive increase of the total exercise performance time in 30 minutes upon the drug administration, as compared to the baseline value of the experimental group at the start of the experiment and the control group. The tests were performed on the $1^{\text {st }}, 8^{\text {th }}, 15^{\text {th }}$ и $22^{\text {nd }}$ day upon the drug administration, and in five days upon the discontinuation of drug administration (on the $27^{\text {th }}$ day), in order to detect the delayed effects of the drugs.

2) For simulating the static physical load, the test of "holding on a vertical grid" was used. The drug potency criterion was a positive increase of the total test performance time (upon five representations) in the static exercise performance conditions in 30 minutes after the nasal administration of the nanodrug, as compared to the control group and to the reference compound of L-DOPA with a similar targeted action.

3) Researching the vestibulo-locomotor activity on the RotaRod device is based on the animal's capability to balance on a rotating cylinder (rod), while the speed of the rod is slowly but continuously accelerated (the initial rod rotation speed was 0.4 $\mathrm{rpm}$, acceleration was $0.4 \mathrm{rpm} / \mathrm{s}$ ). During the experiment, the time was registered, within which the rats managed to stay on the RotaRod (Columbus Instrument, USA). The procedure was repeated three times. Test was conducted in 30 minutes upon drug administration.

4) The behavior of rats in the "open field" test was researched in order to estimate possible consequences of chronic administration of Nano-L-DOPA for the motor and exploratory activity of unrestricted experimental animals within 5 minutes. A special attention was paid to the following parameters: horizontal and vertical activity, number of visits to the center of the “open field”, as well as the number of grooming acts.

The statistical analysis of results obtained was performed using Microsoft Excel 2010 and STATISTICA 8.0.

\section{Results}

1) Chronic nasal administration of the minimum dose under research $(1 \mathrm{mg} / \mathrm{kg})$ of "Nano-L-DOPA" into rats was proved to be positively more efficient on all testing days, as compared to the same dose of L-DOPA ( $\mathrm{p}<0.01$ ), and resulted in the positive increase of the animals' endurance upon 2-week administration ( $\mathrm{p}<0.01$ ), the effect being retained upon discontinuation of the drug within 5 days, unlike L-DOPA, the comparator drug (Figure 1).

The maximum effect was detected on the $15^{\text {th }}$ day of the experiment at administering "Nano-L-DOPA" dosed as $1 \mathrm{mg} / \mathrm{kg}$ and $10 \mathrm{mg} / \mathrm{kg}$, and on the $22^{\text {nd }}$ day of administering it in the dose of $5 \mathrm{mg} / \mathrm{kg}$. In 5 days upon discontinuation, a positive retention of the high endurance level at the minimum doses under research, $1 \mathrm{mg} / \mathrm{kg}$ and $5 \mathrm{mg} / \mathrm{kg}$, was observed (Figure 2). Important note: Using "Nano-L-DOPA" in all doses under research $(1 \mathrm{mg} / \mathrm{kg}, 5 \mathrm{mg} / \mathrm{kg}$ and $10 \mathrm{mg} / \mathrm{kg})$ does not result in performance decrement within at least five days after the drug administration has been discontinued. The dose of $5 \mathrm{mg} / \mathrm{kg}$ (by
L-DOPA) can be considered the optimal dose of "Nano-LDOPA" resulting in a stable, statistically valid increase in the physical endurance of dynamically loaded rats on all testing days, starting from the $15^{\text {th }}$ day of the experiment, at nasal administration.

2) In the conditions of a static physical load (test "holding on a vertical grid") the chronic nasal administration of NanoL-DOPA had resulted in the positive increase of the total time of the rats' holding on the grid as compared to the control group and to the comparator by the $16^{\text {th }}$ day of administering "NanoL-DOPA". The dose-dependent effect of using the nanodrug was observed, the doses of 5 and $10 \mathrm{mg} / \mathrm{kg}$ being most efficient $(\mathrm{p}<0.03$ and $\mathrm{p}<0.01$, respectively). In five days upon the discontinuation of the drugs, the positive differences only retained in the group of animals administered with the nanodrug dosed as $5 \mathrm{mg} / \mathrm{kg}(\mathrm{p}<0.2)$. Using L-DOPA, the comparator drug, resulted in no increase in the endurance within this experiment both during the drug administration and upon its discontinuation.

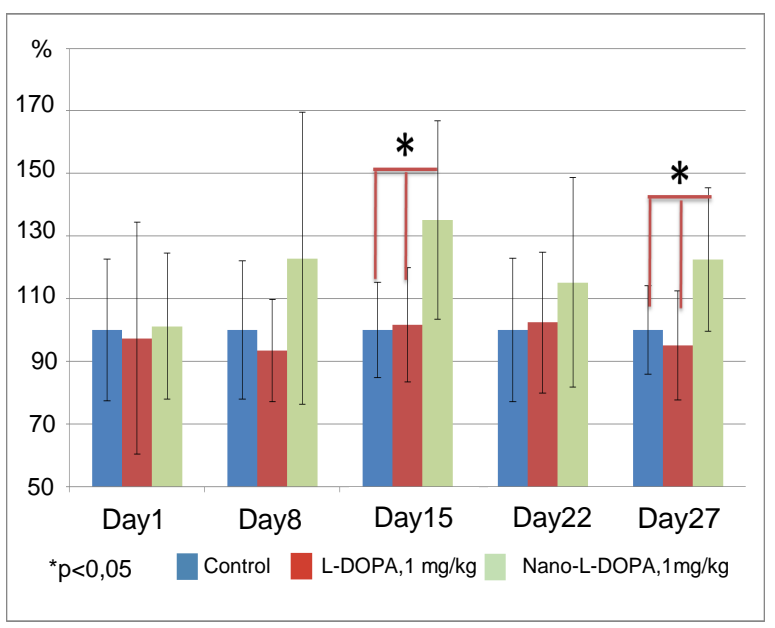

Figure1. Efficacy of "Nano-L-DOPA" as compared to "L-DOPA" at chronic nasal administration of $1 \mathrm{mg} / \mathrm{kg}$ in the test "forced swimming" with weights.

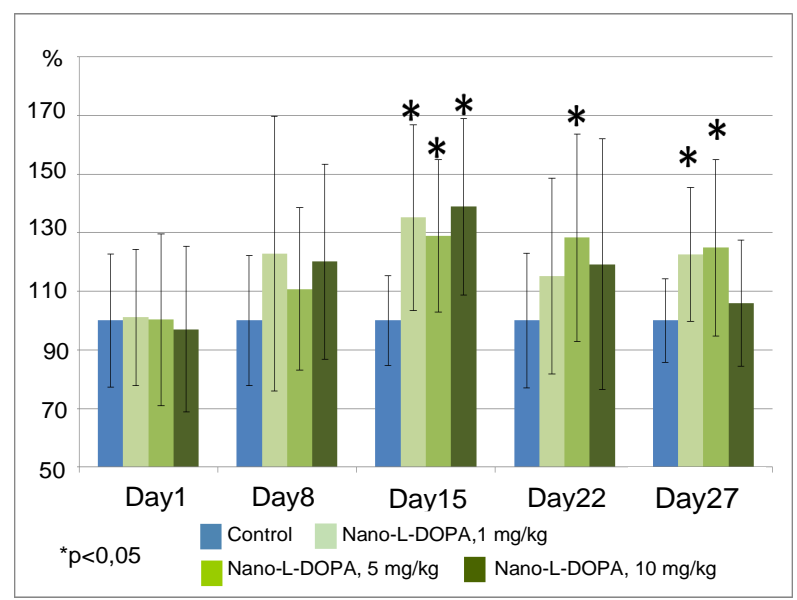

Figure 2. "Nano-L-DOPA" efficacy comparison for different doses at chronic nasal administration in the test "forced swimming" with weights. 
3) The research in the vestibulo-locomotor activity of Wistar rats using RotaRod in the conditions of chronic nasal administration of "L-DOPA" compounds within 20 days, in the minimal daily doses of 1,5 or $10 \mathrm{mg} / \mathrm{kg}$, did not show any disturbances in locomotor activity or any spatial disorientation in the animals. Administering "Nano-L-DOPA" in all doses under research promoted the positive improvement of the animals' rotarod performance and spatial orientation ability.

The largest values of RotaRod performance had reached statistically valid differences in all groups of animals administered with the nanodrug, as compared to the control group ( $>>0.1$ ) and to the group administered with standard medication L-DOPA ( $>0.2$ ), by the end of the experiment (the $20^{\text {th }}$ day of nasal administration). The effect observed was dose-independent and had practically identical representation for all nanodrug doses used. The effect above retained even 6 days upon the nanodrug discontinuation in the groups of animals administered with the doses of 1 or $5 \mathrm{mg} / \mathrm{kg}$. Thus, the identified bettering the coordination of spatial motions and of the animals' spatial orientation ability is the result of using "L-DOPA" in a prolonged form.

4) When studying the drugs influence upon the animals' motor performance and their exploratory activities in the "Open Field” test, the chronic nasal administration of L-DOPA compounds in all doses used was not detected to influence on test results, such as total distance, number of postures and visits to the center of the field. At the same time, it is important to note the disappearance of grooming acts by the end of experiment for all drugs under research, but with the high degree of confidence - in the case of "Nano-L-DOPA" only. This fact may testify the decrease in emotional reactivity/anxiety, as well as the increase in the decision making processes in experimental animals upon the chronic nasal administration of "Nano-LDOPA” and, to a smaller extent, L-DOPA.

Thus, the comprehensive preclinical efficacy of the nasal administration of "Nano-L-DOPA" showed that, in the conditions of a chronic experiment, in the background of the prolonged action of "Nano-L-DOPA", a long-term increase in the animals' endurance under the dynamic and static loads had been detected. Of the doses researched, the optimal doses are 1 $\mathrm{mg} / \mathrm{kg}$ and $5 \mathrm{mg} / \mathrm{kg}$ (by L-DOPA), where the performance increases by maximum (up to $35 \%$ of the background values), the positively significant effect being retained even after the discontinuation of the administration. The results obtained allow us to consider "Nano-L-DOPA" a prospective compound for nasal administration as a drug influencing the performance.

Comparative studies of general toxicity (acute, subacute) at the intranasal administration of the "Nano-L-DOPA" composition, conducted on 2 animal species of both genders (BALB/C mice and Wistar rats) within the doses experimented did not show any toxic actions of "Nano-L-DOPA". In acute toxicity studies, the intranasal (repeated, at short intervals) administration of "Nano-L-DOPA" in a maximum species-relevant volumes and doses was established not to be accompanied by lethal outcomes for the animals under test. The highest summary dose of "Nano-L-DOPA" reached at repeated intranasal administration made $540 \mathrm{mg} / \mathrm{kg}$ in BALB/c mice (by $10 \mathrm{mcl}$ in each nostril, every 5 minutes) and $150 \mathrm{mg} / \mathrm{kg}$ in Wistar rats (by
$20 \mathrm{mcl}$ in each nostril, every 5 minutes), respectively. The same procedure of L-DOPA intranasal administration did not result in any mortality of the animals under test, either. However, transient toxic manifestations were detected at positively smaller doses and were more evident than in administering "Nano-L-DOPA".

In subacute toxicity studies, the 7-day intranasal "Nano-LDOPA" administration in doses under research (rats $20 \mathrm{mg} / \mathrm{kg}$, $50 \mathrm{mg} / \mathrm{kg}$ ) were not accompanied by any lethal outcomes or side effects during the administration period and within 14-day observations after the last administration. The results of hematologic and morphometric studies, pathomorphological and pathohistological examinations did not detect any essential differences in organs and tissues of the animals under test, as compared to those of control animals. The results obtained in the studies allowed us to conclude that the intranasal administration of "Nano-L-DOPA" was a relatively safe way of administering the drug under development. The results obtained allow us to forecast that the intranasal way of administering the "Nano-L-DOPA" will be prospective for further clinical management thereof.

\section{REFERENCES}

[1] A. Pires, A. Fortuna, G. Alves, A. Falcao Intranasal Drug Delivery: How, Why and What for? J Pharm Pharmaceut Sci, 2009, vol. 12(3) pp. 288 - 311

[2] Graff CL, Pollack GM. Nasal drug administration: Potential for targeted central nervous system delivery. J Pharm Sci, 2005, vol. 94, pp. 1187-1195.

[3] Illum L. Transport of drugs from the nasal cavity to the central nervous system. Eur J Pharm Sci, 2000, vol. 11, pp 1-18.

[4] Illum L. Is nose-to-brain transport of drugs in man a reality. J Pharm Pharmacol, 2004, vol. 56, pp. 3-17.

[5] Dahlin M, Jansson B, Bjork E.. Levels of dopamine in blood and brain following nasal administration to rats. Eur J Pharm Sci, 2001, vol. 14. pp. 75-80.

[6] M.Fazil, Shadab, S. Baboota, JK Sahni, J Ali Nanotherapeutics for Alzheimer's disease (AD): past, present and future, Journal of Drug Targeting, 2012, vol. 20 (2), pp. 97-113.

[7] Henry, R.J., Ruano N, Casto D, Wolf RH, A pharmacokinetic study of midazolam in dogs: nasal drop vs. atomizer administration. Pediatr Dent, 1998, vol. 20(5), pp. 321-326.

[8] Sakane, T., Akizuki M, Yoshida M, Yamashita S, Nadai T, Hashida M, Sezaki H. Transport of cephalexin to the cerebrospinal fluid directly from the nasal cavity. J Pharm Pharmacol, 1991, vol. 43(6), pp. 449-451.

[9] Banks, W.A., M.J. During, and M.L. Niehoff Brain uptake of the glucagon-like peptide-1 antagonist exendin (9-39) after intranasal administration. J Pharmacol Exp Ther, 2004, vol. 309(2), pp. 469-475.

[10] Westin, Bostrom E, Grasjo J, Hammarlund-Udenaes M, Björk E. Direct nose-to-brain transfer of morphine after nasal administration to rats. Pharm Res, 2006, vol. 23(3), pp. 565-572.

[11] Kreuter J. Nanoparticulate system for brain delivery of drags. Adv Drug Deliv Rev, 2001, vol. 47, pp. 65-81.

[12] Hall JB, Dobrovolskaia MA, Patri AK, McNeil SE. Characterization of nanoparticles for therapeutics. Nanomedicine (Lond). 2007, Dec, vol. 2(6), pp. 789-803 